PREHOSPITAL CARE

\title{
Passing the Diploma in Immediate Medical Care of the Royal College of Surgeons of Edinburgh
}

\author{
K M Porter, B Steggles
}

Emerg Med J 2005;22:446-449. doi: 10.1136/emj.2004.020321

The Diploma in Immediate Care of the Royal College of Surgeons of Edinburgh is increasingly recognised as a gold standard for immediate care practitioners, including doctors with a significant involvement in prehospital care, paramedics who wish to enhance their knowledge base or obtain the Diploma as an entry requirement for a post registration degree, and for nurses who have a recognised and developing role in the evolving emergency medical services. This paper provides information about the Diploma examination including eligibility, the syllabus and the components of the examination, and comments on the reason for failure and provides suggestions for training and education to enhance the candidate's opportunity of passing the examination.

See end of article for authors' affiliations

Correspondence to: Mr K Porter, Selly Oak Hospital, Selly Oak, Birmingham B29 6JD, UK; keith.porter@uhb.nhs.uk

Accepted for publication 31 March 2005
$\mathrm{T}$ he first diet of the Diploma in Immediate Medical Care (Dip IMC) of the Royal College of Surgeons of Edinburgh was held in 1988. Initially open to qualified medical practitioners, the examination was opened to nurses and paramedics in 1998. The pass rate for this examination is frequently between $50-55 \%$ and although failure may reflect poor underpinning of knowledge, more commonly it is due to poor practical skills and a lack of first hand experience in the area of prehospital care, in particular its demands and limitations. This paper details eligibility, the syllabus and the examination components as well as critically analysing why candidates fail, and offers advice to optimise the candidate's chance of passing the examination.

\section{ELIGIBILITY}

\section{Medical practitioners}

Doctors must have been engaged in the practice of their profession for not less than two years after registration. Candidate must show documented evidence of clinical experience in the area of prehospital emergency care for a period of one year. They must also show evidence of completion of training of not less than three months' full time or equivalent part time in hospital posts approved by the College in the management of the seriously ill or injured patient. This may include participation in a vocational training programme.

\section{Nurses}

Nurses must hold registration with the Nursing and Midwifery Council, or its equivalent and have been engaged in the practice of their profession for not less than two years thereafter. They must also show documented evidence of clinical experience in prehospital emergency care for a period of at least one year and of completion of training of not less than three months in hospital posts approved by the College which includes emergency medicine.

\section{Paramedics}

Paramedics must show evidence of state registration as a paramedic in the United Kingdom (or non-NHS equivalent). Alternatives, such as armed services training may be recognised by the College. Candidates must show documented evidence of clinical experience in the area of prehospital care for a period of 18 months post registration.

Evidence of clinical experience in prehospital emergency care is straightforward for paramedics as this is a major component of their regular work. For doctors and nurses the College recognises that this one year of experience would not be continuous service. Candidates need to provide evidence of spending time with their local ambulance service including time on vehicles with paramedics, a visit to ambulance control, and familiarity with equipment and the major incident procedure. Clinical experience in other avenues of prehospital care-for example, work with the voluntary aid societies, mountain rescue, crowd doctor, motorsport-should be documented and submitted. For doctors, experience in day time and out of hours emergency care provided by general practitioners will compliment the above experience.

Candidates who do not fulfil the normal entry requirement may apply for special consideration and, following submission to the registrar, their curricula vitae and experience will be considered by the Specialty Advisory Board in Immediate Medical Care.

\section{SYLLABUS}

The knowledge base and practical skills necessary to successfully pass the Dip IMC examination include:

\section{Anatomy}

A knowledge of basic anatomy but to include in particular

- the skeletal system with relation to major fractures and soft tissue injuries

- the skull and spine in associated with cranial, maxillofacial, and spinal injuries and knowledge of related neurology 
- the respiratory system including the larynx and trachea

- the cardiovascular system with relation to injuries and myocardial infarction and cardiac arrhythmias

- the abdomen and pelvis with relation to acute emergencies and injury.

\section{Physiology and pathology}

A knowledge in general but to include that relevant to

- the immediate and late effects of severe trauma

- hypovolaemic, cardiogenic, neurogenic, and anaphylactic shock

- cardiopulmonary resuscitation

- respiration to include the effects of chest and "head" injuries

- asphyxiation, drowning, and inhalation of toxic substances

- serious infections and associated risks: control, consequences, and management

- acute metabolic disturbances

- airway obstruction

- airway problems in infancy.

\section{Pharmacology}

A knowledge in general but to include that relevant to

- accidental and non-accidental poisoning, including toxic substances and hazardous chemicals

- analgesia and sedation

- acute medical problems

- acute respiratory problems including asthma

- cardiac arrhythmias and arrest

- intravenous fluids and appropriate indications for their use

- emergency drugs in adults and children, including routes and dosage

- a knowledge of the regulations for prescriptions only medicines for use by medical and paramedical personnel.

\section{Clinical topics}

- the mechanism of injury

- assessment of the scene including risks and hazards

- the clinical signs of severe illness or injury

- the methods and significance of clinical assessment and appropriate recording of observations

- the indications and procedures for resuscitation, including the support of respiration and fluid replacement

- assessment and acute management of injuries to the brain and spinal cord

- the diagnosis and management of myocardial infarction and arrhythmias including thrombolysis

- the acute management of burns

- the acute management of fractures and soft tissue injuries

- the transport of the seriously ill or injured casualties by land, sea, or air.

\section{Practical skills}

- control of haemorrhage and intravenous infusion

- management of the unconscious patient (including related "head" injury)

- management of the airway

- basic airway care
- the use of suction, airway adjuncts, and oxygen

- endotracheal intubation and use of the laryngeal mask airway

- circothyroidotomy

- artificial ventilation

- pneumothorax and haemorthorax, including needle thoracostomy

- management of cardiac arrest

- recognition

- cardiopulmonary resuscitation including defibrillation with manual and automated defibrillators

- knowledge of pacemakers

- management of spinal injuries

- management of limb injuries

- methods for the provision of analgesia and sedation.

\section{Management skills}

- priority in the sorting of casualties including triage sort and sieve

- understanding of the working of the Emergency Services and the integration of medical response

- understanding of principles and methods of communication

- understanding of the problems associated with mass gatherings

- understanding basic principles of major incident management.

\section{GUIDE TO CANDIDATES}

The examination for the Diploma in Immediate Medical Care is designed to test the ability of prehospital practitioners to display their knowledge of emergency medicine to a standard that could be reasonably practiced outside of a hospital. The examination therefore concentrates on the methods of recognition, diagnosis, and management of conditions and situations in the prehospital environment rather than the use of facilities for investigations and treatment that may be available only within a hospital.

For candidates from overseas it is recognised that there are variations from UK practice and procedures in the management of certain conditions. Candidates will not be penalised for this provided they are able to explain their reasoning.

\section{FORMAT OF THE EXAMINATION}

The examination consists of the following components:

(a) a theoretical paper consisting of

(i) a projected material question paper for which 30 minutes is allowed

(ii) a multiple choice paper of 20 questions for which 20 minutes is allowed

(iii) a short answer question paper with six questions for which 30 minutes is allowed

(iv) a written incident scenario exercises for which 15 minutes is allowed.

(b) a practical examination in two parts each of 30 minutes' duration consisting of

(i) a core skills assessment including a general viva examination on all aspects of resuscitation

(ii) a clinical incident scenario and viva examination. The sections of the examination are marked individually but are assessed together to determine a final mark. Candidates who fail the Core Skills part of the examination are deemed to have failed the examination overall. 


\section{Projected material (30 minutes)}

For this part of the examination there are 20 questions; the candidate has 90 seconds to answer each. The first 10 questions are on ECGs-either rhythm strips or 12 lead ECGs. Specific questions are asked on the supporting written paper and marks allotted for the ECG diagnosis and treatment.

An example question might be: "you are called to see a 65 year old man with central chest pain radiating to the jaw and the left arm. There is no associated shortness of breath

(a) What is the ECG diagnosis?

(b) What treatment should be given before transport to hospital?"

The second half of the examination is comprised of slides which include incident scenarios or injuries and equipmentfor example, an RTA (now commonly referred to as a road traffic collision (RTC)) scene, a chemical incident, a compound ankle fracture, or a pulse oximeter.

Questions may relate to scene management, clinical management, or the advantages and disadvantages for the use of a piece of equipment in prehospital care.

\section{Short answer section (30 minutes)}

For this component of the exam the candidate has 30 minutes to answer six questions (five minutes per question) Marks are allocated with reference to a predetermined mark sheet, which gives credit for essential and important aspects of patient care or the whole scenario depending on the question. Sample questions:

- Describe the features and treatment of life threatening asthma in the prehospital situation.

- Describes the uses of adrenaline in prehospital care.

- Describe the problems that may occur in prehospital care following thrombolysis management.

\section{Multiple choice paper (20 minutes)}

The MCQ component of the paper consists of 20 questions for which 20 minutes are allowed. The questions take the form of a statement and five possible answers of which one only is correct. There is no negative marking.

\section{Incident scenario exercise (15 minutes)}

For this part of the examination the candidate is given a clinical scenario which will include information on a number of casualties giving clinical parameters for each patient such that they can be assessed by the triage sieve. As part of the answer the candidate needs to be familiar with incident command, control and communication and resource management and detail how these would be used in the management of the scene. Having undertaken a triage sieve the candidate may be asked to prioritise patients for transportation to hospital.

\section{Core skills (30 minutes)}

The candidate is tested on basic life support and advanced life support in both adults and children. This includes the familiarity with equipment, for example, an oxygen cylinder, airway adjuncts, the equipment for intubation, and the use of the laryngeal mask airway. Additional skills include how to turn a patient to and from the recovery position and to maintain inline stabilisation if appropriate. The candidate should have a reasonable knowledge of resuscitation drugs including dosage and pharmacology and they should be familiar with resuscitation equipment. The candidate may be asked to change an oxygen cylinder. Candidates must pass this section-failure to do so means that they fail the whole examination.

\section{Incident scenario and general viva ( 30 minutes)}

The first part of this examination takes the form of a trauma scenario. The candidate is briefed on the history and has to manage the scene and the patient in a hands-on capacity. Paramedic assistance becomes available and standard equipment for ABCDE treatment is available. The candidate is required to give a formal handover of the patient at the end of the scenario. The simulated casualty is a member of the Casualties Union and the injuries are very realistic.

The second part of this examination is structured question and answer oral session with the examiners covering a number of topics, for example, diabetic coma, status epilepticus, and anaphylaxis. The answers should, where possible, be evidence based, for example, Resuscitation Council guidelines, NICE guidelines, SIGN guidelines, and contemporary consensus publications.

\section{WHY CANDIDATES FAIL}

\section{Projected material question paper}

It is essential to have knowledge of rhythm recognition and the ECG criteria for common diagnoses-for example, VF, VT, SVT, and acute coronary syndromes. Candidates regularly misinterpret infarction patterns and common rhythm irregularities. The supplementary requirement to each question is often inadequately answered, for example, the management of an obvious myocardial infarction should include oxygen therapy, morphine, an antiemetic, aspirin, nitrates, and consideration given to thrombolysis depending on local practice as part of a joint ambulance/doctor response. The hospital should be alerted, careful monitoring undertaken throughout, and the carer prepared to initiate resuscitation in the event of a cardiac arrest.

Candidates fail to read the questions clearly when answering the second half of the paper. The question will clearly state, if appropriate, "scene management", "the management of this patient", or "the management of this injury". When asked about a specific injury-for example, an open fracture dislocation of an ankle-candidates will not get credit for talking about scene safety and airway and breathing assessment. This approach has been taken to ensure candidates do not routinely answer "scene safety and ABCDE assessment" for each question.

Questions relating to pictures showing equipment, for example a pulse oximeter, and asking about its function, indication and contraindications in prehospital practice should be easy marks.

\section{Short answer question paper}

Candidates are often pushed for time in answering this section of the examination. They frequently fail to read the question properly and answer inappropriately. Answers should be in a notation or list format where there are predetermined answers, sometimes key words, for example, "morphine" in acute myocardial infarction or "scene safety" when asked to manage the whole incident score marks. For this reason it is essential the candidate answers every question and includes the essential features of the clinical scenario. Candidates frequently suggest treatment that is only available in hospital-for example, blood gas analysisand some answers fail to recognise the limitation of prehospital care. Some candidates regurgitate "scene safety, ABCDE assessment" for each question when it is not necessary and this reflects a lack of understanding of the question and can frustrate the examiner.

\section{Multiple choice paper}

Adequate time is allocated for this section and poor results usually reflect a lack of basic knowledge. 


\section{Written incident scenario exercise}

Candidates frequently fail to demonstrate basic knowledge of the management of a major accident scene. Candidates should be familiar with the triage sieve and sort and should be able to prioritise patients for treatment and evacuation. This component of the examination follows the short answer question paper and some candidates fail to complete this section because they have devoted too much time to the SAQs. As a consequence they may receive a low mark on this section, which may determine an overall failure when the accumulative marks are reviewed.

\section{Core skills station}

This section requires knowledge of the Resuscitation Council algorithms including arrest and peri-arrest scenarios. Candidates regularly fail to follow the algorithms, to demonstrate appropriate airway care, and have poor defibrillator safety. This practical section of the examination is the same for each candidate every time and is marked to a standard checklist. Candidates are strongly advised to obtain practical training and assessment and coaching either with hospital trusts, ambulance services, or other appropriate organisations to ensure their competence before the examination.

\section{Clinical incident scenario}

Candidates fail this component of the examination because of a lack of prehospital care experience, real time or moulage practice, and a lack of familiarity with standard equipment. Appropriate allowance is given to candidates from countries where there may be a difference in emergency care provision and equipment. "Patients" are members of the Casualty Union and have very realistic injuries and may complain to the examiner about a lack of communication or poor handling by the candidate. A number of candidates fail to demonstrate practical competence-for example, in-line manual stabilisation of the cervical spine or use of equipment (for example sizing and applying a cervical collar). Handover to the ambulance service or in hospital is often nonstructured and incomplete.

The second part of this section involves a structured viva examination and should be straightforward, providing the candidate has a satisfactory clinical knowledge and can relate assessment and treatment to the prehospital environment.

\section{RECOMMENDED READING AND COURSES}

The College of Surgeons has a recommended reading list, which is supplied to candidates with the examination application form and guidance document. Candidates are strongly recommended to be familiar with the latest guidelines, for example, Resuscitation Council guidelines and the British Thoracic Society Asthma guidelines. The prehospital care section of the Emergency Medicine Journal (EMJ) provides important information including evidence based papers and consensus papers of relevance. The interface with emergency medicine should be seamless and much of the rest of the content of the EMJ will be relevant reading for the Diploma candidate.

The British Association for Immediate Care (BASICS) runs an excellent five day course with a proven track record of candidates passing the Diploma. In addition the Department of Academic Medicine at the University of Teeside has recently introduced a crammer course which includes how to pass the exam, as well as the necessary academic and practical skills.

\section{SUMMARY}

The Diploma in Immediate Medical Care examination is increasingly recognised as a gold standard in prehospital care. The examination requires an in-depth theoretical and practical knowledge, the latter of which is best gained by experience working with the ambulance service. The BASICS course has a proven track record in providing the necessary hands on practice and moulage experience necessary to pass this examination. The College offers advice to candidates and can help to facilitate practical training and clinical experience. Increasingly, the Diploma in Immediate Medical Care is becoming a requirement for certain medical roles and a post registration entry requirement for paramedics for some degree courses.

Further information on the Diploma can be found at http:// www.rcsed.ac.uk
Authors' affiliations
K M Porter, Honorary Secretary, Faculty of Prehospital Care, Royal
College of Surgeons of Edinburgh, Edinburgh, UK
B Steggles, Chairman, Faculty of Prehospital Care, Royal College of
Surgeons of Edinburgh, Edinburgh, UK 